\title{
Candidiasis invasoras en recién nacidos: diagnóstico, tratamiento y prevención
}

\author{
Giannina Izquierdo y María Elena Santolaya
}

\section{Invasive candidiasis in newborns: diagnosis, treatment and prophylaxis}

Invasive fungal infections have become an increasingly common problem in neonatal intensive care units (NICU). Invasive candidiasis (IC) is associated with substantial morbidity and mortality rates, especially in preterm infants. The aim of this review is to suggest actions in monitoring, prevention, treatment and follow up of $\mathrm{IC}$ in the newborn infant.

Key words: Candida, candidemia, invasive candidiasis, newborn, neonatal intensive care unit.

Palabras clave: Candida, candidemia, candidiasis invasora, unidad de cuidados intensivos neonatales.

\section{Introducción}

\section{$\mathrm{L}$} a incidencia de candidiasis invasoras (CI) en las unidades de cuidados intensivos neonatales (UCIN) ha aumentado considerablemente en las últimas décadas, debido al aumento en la supervivencia de los recién nacidos $(\mathrm{RN})$ prematuros y de bajo peso de nacimiento, asociado a cambios en los procedimientos diagnósticos y terapéuticos. La frecuencia de CI es variable dependiendo del centro y de la complejidad de éste. Los datos existentes se basan en estudios multicéntricos, principalmente norteamericanos, con limitados reportes sobre la incidencia de CI en Latinoamérica ${ }^{1,1 a}$, por lo que parece fundamental que cada UCIN evalúe cuál es la tasa de incidencia de infecciones invasoras por Candida spp, con el objetivo de establecer una adecuada estrategia local de vigilancia, prevención, diagnóstico y manejo.

El objetivo de este documento es sugerir acciones de vigilancia, prevención farmacológica, diagnóstico, tratamiento y seguimiento de las CI en RN.

\section{Definiciones}

- Recién nacido pre-término de muy bajo peso de nacimiento (RNpret MBPN): $<32$ semanas y/o $<1.500 \mathrm{~g}^{2}$.

- Recién nacido pre-término extremo (RNpret extremo): $<1.000 \mathrm{~g}^{2}$.

- Candidiasis invasora (CI): Aislamiento de Candida spp desde un sitio estéril, siendo la sangre (candidemia) el principal lugar de aislamiento (70\%); le siguen en frecuencia: orina (candiduria) (15\%) (por punción suprapúbica con una colonia o con cateterismo vesical, con un recuento $>$ de $10.000 \mathrm{ufc} / \mathrm{ml}$ ), líquido céfaloraquídeo (LCR) $(10 \%)$ y otros fluidos corporales (líquido peritoneal, articular, etc) (5\%).

- Candidemia persistente: Persistencia de hemocultivos
(HCs) positivos a las $72 \mathrm{~h}$ del inicio de un tratamiento antifúngico efectivo. Ocurre en 10-30\% de las infecciones invasoras por Candida $\mathrm{spp}^{3-5}$.

\section{Epidemiología}

Según datos obtenidos de literatura anglosajona, Candida spp es el principal microorganismo causal de micosis oportunistas en el medio intrahospitalario. En el grupo de RNpret MBPN Candida albicans ocupa el tercer lugar como agente causante de sepsis neonatales tardías, definidas como aquellas que ocurren después de las $72 \mathrm{~h}$ de vida, luego de Staphylococcus coagulasa negativa y Staphylococcus aureus ${ }^{6}$. En neonatos, C. albicans ocupa $58 \%$ de los aislados en sangre; sin embargo, la frecuencia de otras especies como Candida parapsilosis (34\%) ha ido en aumento en los últimos 10 años. Menos frecuente es el aislamiento de Candida glabrata (4\%) y Candida tropicalis $(2 \%)^{6}$.

La incidencia de CI es inversamente proporcional al peso de nacimiento, comunicándose cifras de 2,6 a $16,7 \%$ en RNpret MBPN y superiores a $20 \%$ en RNpret extremo $^{7,8}$.

La CI en RNpret extremo presenta una mortalidad de $20 \%$ a pesar del tratamiento antifúngico apropiado y hasta $60 \%$ de ellos desarrollan secuelas neurológicas significativas 9 .

La principal fuente de infección ocurre desde la colonización cutánea y gastrointestina $1^{10}$. Se presume que $5 \%$ de los RN presenta colonización gastrointestinal al momento de ingresar a una UCIN, llegando a 50\% en la primera semana y $75 \%$ al mes de vida. La principal fuente de adquisición de C. albicans es el canal del parto, a diferencia de $C$. parapsilosis que se asocia en mayor
Facultad de Medicina, Universidad de Chile, Santiago. Departamento de Pediatría y Cirugía Infantil, Campus Oriente, Hospital Dr. Luis Calvo Mackenna, Santiago, Chile.

Programa de Formación en Infectología Pediátrica (GI) Unidad de Infectología Pediátrica (MES)

Recibido: 14 de agosto de 2013 Aceptado: 25 de noviembre de 2013

M. Elena Santolaya es miembro de la Red Latinoamericana de Infecciones Fúngicas Invasoras, proyecto financiado por Pfizer Inc.

No existió financiamiento específico para la realización de este trabajo.

Correspondencia a:

Giannina Izquierdo Copiz gianninai@yahoo.es 
Tabla 1. Resultados de la vigilancia de candidemias en menores de 18 años de la Red Latinoamericana de Enfermedades Fúngicas Invasoras. Distribución por especies de Candida spp. en RN y niños

$\begin{array}{lccc} & \begin{array}{c}\text { Recién nacidos } \\ \mathbf{n}(\mathbf{\%})\end{array} & \begin{array}{c}\text { Niños } \\ \mathbf{n} \mathbf{( \% )}\end{array} & \begin{array}{c}\text { Total } \\ \mathbf{n}=\mathbf{3 0 2}\end{array} \\ \text { C. albicans } & 39(43,8) & \mathbf{n = 2 1 3} & 115(38,1) \\ \text { C. parapsilosis } & 24(27,0) & 76(35,7) & 80(26,5) \\ \text { C. tropicalis } & 13(14,6) & 56(26,3) & 44(14,6) \\ \text { C. guilliermondii } & 4(4,5)^{*} & 31(14,6) & 31(10,3) \\ \text { C. glabrata } & 3(3,4) & 27(12,7)^{*} & 10(3,3) \\ \text { C. } \text { krusei } & 4(4,5) & 7(3,3) & 9(3,0) \\ \text { Otras } & 2(2,2) & 5(2,3) & 13(4,2)\end{array}$

*Valor de $P$ no significativo para todas las especies, excepto para $C$. guilliermondii $(P=0,03)$. †Otras especies de Candida: Neonatos: C. lusitaniae (1), C. intermedia (1). Niños: C. haemulonii (3), C. pelliculosa (3), C. intermedia (2), C. norvegiensis (1), C. lusitaniae (1) , C. albicans + C. glabrata (1).

Tabla 2. Distribución por especies de Candida en hemocultivos y susceptibilidad antifúngica. Hospital Luis Calvo Mackenna (noviembre 2008 - febrero 2013)

$\begin{array}{lccccc}\text { Candida spp } & \mathbf{n} & \% & \text { Fluconazol } & \text { Voriconazol } & \text { Anfotericina B* } \\ \text { C. albicans } & 13 & 44,8 & \mathrm{~S} & \mathrm{~S} & \mathrm{~S} \\ \text { C. parapsilosis } & 9 & 31 & \mathrm{~S} & \mathrm{~S} & \mathrm{~S} \\ \text { C. tropicalis } & 3 & 10,3 & \mathrm{~S} & \mathrm{~S} & \mathrm{~S} \\ \text { C. guilliermondii } & 2 & 6,9 & \mathrm{~S}-\mathrm{R}^{\text {\& }} & \mathrm{S} & \mathrm{NR} \\ \text { C. glabrata } & 1 & 3,5 & \mathrm{R} & \mathrm{S} & \mathrm{NR} \\ \text { C. krusei } & 1 & 3,5 & \mathrm{~S}^{* *} & \mathrm{~S} & \mathrm{NR} \\ \text { C. lusitaniae } & 0 & 0 & - & - & \mathrm{NR} \\ & 29 & 100 & & & \end{array}$

*Estudio de susceptibilidad a anfotericina B realizado desde mayo 2012. ** Sensible dosis dependiente. \&1 cepa $\mathrm{S}$ y una cepa R. NR: No realizado.

\section{Tabla 3. Factores de riesgo de candidiasis invasora}

$\begin{array}{llll}\begin{array}{l}\text { Edad gestacional } \\ \text { y peso nacimiento } \\ <28 \text { sem }\end{array} & \begin{array}{l}\text { Medicamentos } \\ \text { Antimicrobianos de amplio } \\ \text { espectro: Cefalosporinas } \\ \text { de } 3^{\mathrm{a}} \circ 4^{\mathrm{a}} \text { generación } \\ \text { Carbapenémicos }\end{array} & \text { ECN } & \text { Otros factores } \\ <1.000 \mathrm{~g} & \begin{array}{l}\text { Bloqueadores } \mathrm{H} 2 \\ \text { Inhibidores de bombas de } \\ \text { protones }\end{array} & \begin{array}{l}\text { Perforación focal } \\ \text { intestinal }\end{array} & \text { TET } \\ & \begin{array}{l}\text { Enfermedades intestinales } \\ \text { complicadas que requie- } \\ \text { ren ayuno }>7 \text { días* }\end{array} & \begin{array}{l}\text { Colonización } \\ \text { múltiple } \geq 3 \text { sitios }\end{array} \\ & \begin{array}{l}\text { Candidiasis cutánea } \\ \text { congénita }\end{array} & \\ & & & \end{array}$

CVC: Catéter venoso central, TET: tubo endotraqueal, ECN: Enterocolitis necrosante, Bloqueadores H2: antagonistas de receptores de histamina. *Gastrosquisis, onfalocele, atresia intestinal, fístula traqueoesofágica, E. Hirshprung, perforación intestinal espontánea. Modificado de Neonatal candidiasis: Clinical manifestations, management, and prevention strategies. Kaufman D. J Pediatr 2010; 156: S47-52 medida a una transmisión horizontal ${ }^{11}$. Se han comunicado brotes de CI por $C$. parapsilosis en UCIN, secundarios a la transmisión por las manos del personal ${ }^{12,13}$.

La Red Latinoamericana de Enfermedades Fúngicas Invasoras, un grupo formado por médicos infectólogos, microbiólogos, micólogos, oncólogos y especialistas en trasplantes, pertenecientes a 23 hospitales de 8 países de la región, ha desarrollado guías para el diagnóstico y manejo de la CI en RN, niños y adultos ${ }^{14,15}$. Se realizó un estudio de vigilancia entre noviembre 2008 y octubre de 2010 , con el objetivo de conocer la epidemiología de las candidemias en nuestro continente. Durante el período de estudio se registraron 302 episodios de candidemia en pacientes bajo 18 años de edad, 89 de ellos (29\%) en RN ( $\leq 28$ días). La incidencia de candidemia en niños fue de $0,81 / 1.000$ egresos hospitalarios. Las principales especies aisladas en neonatos fueron C. albicans (43,8\%), C. parapsilosis (27\%) y C. tropicalis $(14,6 \%)$; con muy baja frecuencia de Candida guilliermondii (4,5\%), Candida krusei (4,5\%) y C. glabrata $(3,4 \%)$ (Tabla 1$)$.

Con estos datos como base, se realizó una vigilancia activa de candidemia en un período de observación de 4 años y 3 meses (noviembre 2008-febrero 2013) con el objetivo de conocer la epidemiología de las candidemias en el Hospital Luis Calvo Mackenna (HLCM), uno de los hospitales chilenos que formó parte de la vigilancia latinoamericana. En este estudio se registraron 29 episodios de candidemia en el período observado, con una incidencia de 0,56/1.000 egresos hospitalarios. La UCIN del HLCM, que no cuenta con maternidad, ingresa pacientes prematuros derivados de otros centros, en especial para resolución de patología quirúrgica. De los 29 episodios de candidemia en el período de 54 meses analizado, sólo un episodio se presentó en un prematuro extremo de 25 semanas y 660 gramos con múltiples factores de riesgo de CI.

En nuestra realidad, C.albicans representa $44,8 \%$ y C.parapsilosis $31 \%$ de los aislados de Candida spp en muestras de HCs (Tabla 2).

\section{Factores de riesgo}

Los RNpret de MBPN y RNpret extremos presentan una mayor susceptibilidad a infectarse por Candida spp, condición atribuible a la inmadurez de su sistema inmune, al empleo de procedimientos invasores prolongados- ventilación mecánica (VM), nutrición parenteral (NP), catéter venoso central (CVC)- a la transmisión cruzada a través de las manos del personal de salud, a la exposición a diversos medicamentos que favorecen el sobrecrecimiento de hongos (antimicrobianos de amplio espectro, corticosteroides postnatales, antiácidos) y a las probables complicaciones gastrointestinales presentes en este grupo (enterocolitis necrosante, entre otras) (Tabla 3). 
La colonización por Candida spp se considera un factor de riesgo de progresión a CI. El porcentaje de RNpret extremos colonizados que presentan una CI varía entre 7 y $28 \%$ en diferentes series ${ }^{16,17}$. Manzoni y cols., comunicaron una tasa de progresión a CI de $27,5 \%$, en los pacientes colonizados por Candida spp en tres o más sitios versus 7,2\% en aquellos RNpret extremos no colonizados $(\mathrm{p}<0,001)$. No hubo diferencias significativas en la progresión a CI en aquellos RN con colonización de un sitio $^{18}$. La mediana de tiempo en que se desarrolló la CI fue a los 21 días de vida ${ }^{19}$.

Existen escasos datos de colonización por Candida spp en neonatos en nuestro país. En un estudio prospectivo realizado en la UCIN del Complejo Hospitalario San Borja-Arriarán, se detectó colonización, de al menos un sitio, en 13,6\% RNpret MBPN. La colonización rectal fue la más frecuente y en el período de tiempo observado no hubo progresión a candidemia ${ }^{20}$.

Existe otro grupo de pacientes que, sin ser prematuros extremos, presentan también un riesgo elevado de CI; son los pacientes con patología quirúrgica abdominal (gastroquisis, onfalocele, atresia intestinal, fístula traqueoesofágica, enfermedad de Hirchprung complicada, entre otras) que requieren ayuno prolongado ( $>7$ días) y apoyo de NP. Se ha descrito la translocación de Candida spp y su paso al torrente sanguíneo, especialmente desde el tracto gastrointestinal inmaduro y las zonas de anastomosis quirúrgicas recientes, como factores predictores independientes de $\mathrm{CI}^{21}$.

\section{Manifestaciones clínicas}

Las manifestaciones clínicas de CI en el neonato son inespecíficas y sutiles e incluyen intolerancia alimentaria, distensión abdominal, letargia, hipotermia, dificultad respiratoria, apneas, e inestabilidad hemodinámica; por lo que se debe sospechar una CI en aquellos pacientes con factores de riesgo, que presenten manifestaciones clínicas similares a las de una sepsis bacteriana. La presencia de fiebre es poco frecuente, incluso en enfermedad sistémica. Se ha descrito hiperglicemia asociada a trombocitopenia en infecciones fúngicas diseminadas ${ }^{11}$.

Los principales órganos blanco de CI son el tracto genitourinario, globo ocular, sistema nervioso central (SNC), hígado, bazo, corazón, tejido celular subcutáneo, aparato locomotor y pulmón. Las manifestaciones clínicas son variables dependiendo de cada zona afectada.

\section{Diagnóstico}

Frente a la sospecha clínica de CI o frente a la presencia de un HC positivo a Candida spp es mandatorio iniciar una búsqueda de enfermedad fúngica invasora (EFI).
Estudio de una potencial EFI:

- Examen clínico detallado, incluido fondo de ojo

- Exámenes generales:

- Hemograma con recuento de plaquetas (significativo: valor $\left.<100.000 / \mathrm{mm}^{3}\right)^{22}$.

- Glicemia: la presencia de hiperglicemia de reciente comienzo sugiere infección por Candida spp. ${ }^{11}$.

- Estudio de función renal y hepática, previo al uso de antifúngicos.

- Estudio microbiológico:

- Hemocultivos. Se deben tomar dos HCs para obtener un mayor rendimiento del examen. La sensibilidad diagnóstica es baja por lo que tener un $\mathrm{HC}$ negativo no descarta la presencia de Candida spp. Si hay CVC, tomar una muestra a través de esta vía y una por punción periférica. La modalidad de cultivo recomendada es el HC automatizado, idealmente en botellas especiales para RN, y un volumen óptimo de $1 \mathrm{cc}$ de sangre por cada botella. Se diagnostica una candidemia con el aislamiento de Candida spp en al menos un HC, ya sea central o periférico. Si se aísla Candida spp de un HC de CVC, se considera una infección asociada al dispositivo y éste debe ser retirado a la brevedad.

- Orina completa, urocultivo y búsqueda de pseudohifas en una muestra de orina obtenida por cateterismo vesical (otras técnicas de extracción de la orina tienen alto riesgo de contaminación por levaduras genitales/ perineales). Un cultivo de orina positivo se define como la presencia de $>10^{4} \mathrm{ufc} / \mathrm{ml}$ de Candida spp.

- Estudio de líquido cefalorraquídeo (LCR). Se recomienda efectuar tinción de Gram, citoquímico y cultivo con siembra en agar sangre y Sabouraud. Un análisis citoquímico de LCR normal no descarta el compromiso parenquimatoso cerebral por lo que se necesita una neuroimagen complementaria (ver imágenes).

- Examen micológico directo y cultivo de cualquier zona afectada según la evaluación clínica (líquido articular, líquido peritoneal, tejido obtenido por biopsia).

- Las técnicas de diagnóstico molecular y determinación de antígenos están bajo evaluación en RN para complementar el diagnóstico de $\mathrm{CI}^{23,24}$.

- Estudio de susceptibilidad in vitro a toda cepa de Candida spp que sea aislada. Este es de fundamental importancia para el ajuste de la terapia antifúngica. (Tabla 4).

- Imágenes:

- Ecografía cerebral: puede revelar la presencia de dilatación de ventrículos, calcificaciones o bolas fúngicas. En ocasiones, la interpretación puede ser dificultosa en $\mathrm{RN}$ con hemorragia intracraneal o leucomalacia periventricular. La tomografía axial computada (TAC) y la resonancia magnética $(\mathrm{RM})$ muestran ciertas ventajas sobre la ecografía cerebral en el diagnóstico de abscesos cerebrales (con medio de contraste) y 


\begin{tabular}{|c|c|c|}
\hline Medicamento & Dosis & Comentarios \\
\hline Anfotericina B deoxicolato & 0,5 - $1 \mathrm{mg} / \mathrm{kg} / \mathrm{día}$ IV & En 2 a $4 \mathrm{~h}$ \\
\hline Anfotericina B liposomal & 3 - $5 \mathrm{mg} / \mathrm{kg} / \mathrm{día}$ IV & \\
\hline Caspofungina & $25 \mathrm{mg} / \mathrm{mt}^{2} / \mathrm{día}$ IV & $\begin{array}{l}\text { Sólo después de descartar } \\
\text { compromiso de SNC }\end{array}$ \\
\hline Micafungina & $\begin{array}{l}10 \mathrm{mg} / \mathrm{kg} / \mathrm{día} \text { IV } \\
\text { Considerar } 15 \mathrm{mg} / \mathrm{kg} / \mathrm{día} \text { en } \\
\text { compromiso de SNC }\end{array}$ & No disponible aún en Chile \\
\hline Fluconazol & $\begin{array}{l}12 \mathrm{mg} / \mathrm{kg} / \mathrm{dí} \text { IV } \\
\text { Considerar dosis de carga de } \\
25 \mathrm{mg} / \mathrm{kg}\end{array}$ & $\begin{array}{l}\text { Sólo en caso de ITU y paciente } \\
\text { clínicamente estable } \\
\text { No usar en caso de profilaxis } \\
\text { con fluconazol }\end{array}$ \\
\hline
\end{tabular}

calcificaciones; además la RM permite una mejor visualización de la fosa posterior. La ventaja de la ecografía es su fácil acceso, la ausencia de radiación ionizante, la posibilidad de realizar el examen sin sacar al RN gravemente enfermo de la UCIN y el hecho de no requerir sedación/anestesia ${ }^{11}$.

- Ecografía renal y abdominal: su utilidad es localizar bolas fúngicas, definir el compromiso parenquimatoso renal, hepato-esplénico o peritoneal.

- Ecocardiograma transtorácico: el compromiso cardíaco con vegetaciones se describe en 5 a $15 \%$ de los casos

\section{Tratamiento}

A la hora de elegir el tratamiento de una CI se deberá valorar la localización y extensión de la infección, la susceptibilidad in vitro del aislado, el mecanismo de acción y la toxicidad relacionada con el fármaco, así como también se deberá considerar el uso previo de profilaxis medicamentosa.

El tratamiento de una CI en RN debe iniciarse con un antifúngico con actividad fungicida, siendo indicaciones aprobadas anfotericina B deoxicolato (anfo B-d), anfotericina de formulación lipídica (anfo-L) y equinocandinas: caspofungina, micafungina. (Tabla 4).

Anfo B-d por años ha sido el fármaco de elección en el tratamiento de la CI en el RN, teniendo menores efectos adversos que en niños mayores o adultos ${ }^{25}$. Es importante recalcar que la penetración de anfo B-d al LCR en neonatos supera al 40-90\% de las concentraciones encontradas en plasma ${ }^{26}$.

Las reacciones adversas descritas con anfo B-d son nefrotoxicidad, (hipokalemia, hipomagnesemia y, en grado extremo, insuficiencia renal,), mielosupresión y elevación de enzimas hepáticas. La mayoría de las reacciones adversas son dosis dependiente, y reversibles al suspender el tratamiento. Se ha sugerido que existe una menor frecuencia de compromiso renal en la población pediátrica, en especial en los prematuros extremos, lo que se debería a una mayor depuración del compuesto que en los adultos. Sin embargo, esos resultados no son concluyentes $^{27,28}$. Lee y cols., describen una frecuencia de nefrotoxicidad de $16 \%$, definida como aumento de creatininemia $>$ a $0,4 \mathrm{mg} / \mathrm{dl}$ y frecuencia de hipokalemia de $17 \%{ }^{29}$. Las reacciones asociadas a la infusión de anfo B-d (calofríos, fiebre, temblores) tienen menor frecuencia que la descrita en adultos y niños mayores ${ }^{11}$.

Se recomienda que mientras dure el tratamiento antifúngico debe monitorizarse estrechamente la función renal y electrolitos plasmáticos, al menos dos veces por semana $^{28}$.

Las formulaciones lipídicas de anfotericina B como anfotericina liposomal (L-amB), anfotericina de complejo lipídico (ABCL) y anfotericina de dispersión coloidal (ABDC), son una alternativa a anfo B-d, con similar espectro de acción y menor nefrotoxicidad. La FDA aprobó el uso de L-amB (AmBisome ${ }^{\circledR}$ ) para el lactante mayor de un mes de vida, ABCL (Abelcet ${ }^{\circledR}$ ) sobre los 16 meses y ABDC (Amphotec ${ }^{\circledR}$ ) para niños mayores y adultos ${ }^{30}$. La mayoría de los estudios disponibles en la literatura médica, sobre el uso comparativo de anfo B-d y formulaciones lipídicas de anfotericina corresponden a publicaciones de series de casos o trabajos retrospectivos ${ }^{31-33}$. Los ensayos prospectivos se han hecho en base a $\mathrm{L}$-amB donde la tasa de respuesta a tratamiento es comparable con anfo B-d (84 y $89 \%$, respectivamente) $)^{34}$. Con el uso de L-amB no se describen efectos adversos significativos, salvo hipokalemia leve que se recupera pronto con el aporte de potasio $^{35-37}$. Un grupo de expertos de la Sociedad Europea de Microbiología Clínica e Infectología (ESCMID) se refiere al uso de L-amB, en dosis de 2,5 a $7 \mathrm{mg} / \mathrm{kg} /$ día en $\mathrm{RN}$ con un adecuado perfil de seguridad ${ }^{38}$. Los estudios con $\mathrm{ABCL}$ y $\mathrm{ABDC}$ son escasos ${ }^{39,40}$. No está recomendado el uso de formulaciones lipídicas de anfotericina $\mathrm{B}$ en caso de urocultivos positivos a Candida spp. debido a la mala penetración de éstas en el tejido renal ${ }^{30}$.

Las equinocandinas (caspofungina o micafungina), también están recomendadas en el tratamiento de las CI en neonatos. En una experiencia, caspofungina demostró tener mayor eficacia que anfo B-d en el tratamiento de CI. $(86,7 \text { vs } 41,7 \%)^{41}$. En un estudio realizado por SáezLlorens y cols., se midió la concentración plasmática y perfil de seguridad de caspofungina en lactantes bajo 3 meses de edad. Los resultados establecieron que con una dosis de $25 \mathrm{mg} / \mathrm{m}^{2} /$ día IV se alcanzan concentraciones plasmáticas equivalentes a la dosis usual de $50 \mathrm{mg} / \mathrm{m}^{2} /$ día $^{42}$. Caspofungina es bien tolerada, sin efectos adversos significativos, pero está contraindicado por ahora su uso cuando existe compromiso de SNC, debido a la ausencia de estudios concluyentes que evalúen la eficacia del tratamiento en este grupo etáreo. Se ha intentado el 
uso de dosis más altas de caspofungina para alcanzar concentraciones adecuadas en LCR, aún sin resultados suficientes que permitan realizar una recomendación ${ }^{43,44}$.

Micafungina tiene similar eficacia y seguridad que L-amB en pacientes pediátricos (incluidos neonatos) con $\mathrm{CI}^{45}$. Las dosis recomendadas en neonatos se basan en estudios de farmacocinética, donde se observó que este grupo etáreo presenta un mayor volumen de distribución y una rápida excreción del fármaco, haciendo recomendable una mayor dosificación $(10 \mathrm{mg} / \mathrm{kg})$ que en la población pediátrica (2 a $4 \mathrm{mg} / \mathrm{kg})^{46}$. Es la única equinocandina recomendada para utilizar en infecciones del SNC, elevando sus dosis hasta $15 \mathrm{mg} / \mathrm{kg}$ con lo que se logran concentraciones fungicidas en LCR y un adecuado perfil de seguridad ${ }^{47,48}$. A la fecha de la escritura de este documento, micafungina no está disponible aún en Chile ${ }^{49,50}$.

Anidulafungina, efectiva y bien tolerada en niños y adultos, aún existen escasos reportes de su uso en $\mathrm{RN}$, y no está aprobada por la FDA ni por la ESCMID para su uso general en esta edad ${ }^{38,51} \mathrm{ni}$ en particular en el grupo con compromiso del SNC.

Fluconazol, por su efecto fungistático, debe reservarse sólo para RN con candidiasis urinaria, con estudio de diseminación hematógena negativo, lo que es recomendado gracias a su eliminación por vía renal y buena concentración en orina, o para completar tratamiento en $\mathrm{RN}$ con CI que tenga una condición clínica estable y cuya Candida spp sea susceptible a fluconazol (Tabla 5). Si bien la gran mayoría de las especies de Candida spp. son susceptibles in vitro a fluconazol, se debe considerar que C. krusei es intrínsecamente resistente al fármaco, al igual que 30 a $65 \%$ de los aislados de C. glabrata $^{(25)}$ por lo que es fundamental conocer la susceptibilidad del agente aislado. La dosis de fluconazol recomendada para el tratamiento de la candidiasis urinaria es de $12 \mathrm{mg} / \mathrm{kg}$ $\mathrm{IV}^{14}$. Se ha considerado la prescripción de fluconazol con dosis de carga inicial de $25 \mathrm{mg} / \mathrm{kg}$ IV en pacientes críticamente enfermos, para alcanzar concentraciones terapéuticas en forma precoz, pero aún faltan más estudios farmacocinéticos para recomendar su uso masivo en neonatos ${ }^{52,53}$.

En el caso de una CI, es de vital importancia el inicio de un tratamiento antifúngico con un fármaco fungicida, precoz, y retirar el(los) CVCs si lo(s) hay. El retiro precoz de CVC (antes de 24 h) está asociado a menor mortalidad, menor tasa de diseminación, menor tasa de candidemia persistente y mejor desarrollo psicomotor ${ }^{8}$. Este retiro debe ser evaluado en el contexto de los mencionados riesgos de su permanencia versus la falta de disponibilidad de otro acceso vascular, situación frecuente en neonatos.

El tiempo de tratamiento de las CI va a depender de la localización/diseminación de la infección, de la respuesta clínica al tratamiento y de la negativización de los cultivos. Por esto es fundamental un seguimiento clínico y microbiológico estricto en cada caso. En promedio, el tratamiento se debe prolongar por 14 a 21 días después de obtener al menos dos HCs consecutivos negativos. Se ha descrito que hasta en $20 \%$ de los casos, puede haber un $\mathrm{HC}$ negativo seguido de $\mathrm{HCs}$ positivos $^{8}$. En otras palabras, un $\mathrm{HC}$ negativo no garantiza que ese hallazgo se mantenga en el tiempo y es necesario tener al menos dos HCs negativos consecutivos para suspender la terapia antifúngica.

Existen limitados datos en RN que avalen la utilidad de asociar un segundo antifúngico a la terapia ${ }^{35}$.

\section{Tratamiento empírico}

El tratamiento empírico de las CI es controversial. No hay estudios controlados sobre este tema. Se debe considerar la incidencia de CI en cada UCIN y además los factores de riesgo presentes en cada caso.

Un estudio retrospectivo de tratamiento empírico con antifúngicos en RNpret MBPN, gravemente enfermos, con factores de riesgo de infección fúngica, concluyó que la mortalidad relacionada a CI disminuyó significativamente ${ }^{51,54}$.

\section{Tratamiento de las complicaciones}

\section{Endocarditis}

Existen escasos reportes de endocarditis fúngica en RN y la mayoría son comunicaciones de $\operatorname{casos}^{55}$. Los factores predisponentes son: candidemia persistente, VM, antibacterianos de amplio espectro y uso de catéter umbilical. La ubicación más frecuente de la vegetación es la aurícula derecha. El tratamiento es prolongado, 4 a 6 semanas o más, dependiendo de la evolución clínica y ecocardiográfica. Los medicamentos de elección son equinocandinas o anf-L. Requiere de evaluación por cirujano cardiovascular para su eventual cirugía. Es imprescindible retirar el CVC si lo hay.

\section{Tabla 5. Patrones de susceptibilidad de Candida spp. a antifúngicos}

\begin{tabular}{|c|c|c|c|c|}
\hline Candida spp & Anfotericina B & Fluconazol & Voriconazol & Equinocandinas \\
\hline C. albicans & $S$ & $S$ & $S$ & $S$ \\
\hline C. parapsilosis & S & $S$ & $S$ & $\mathrm{Sal}{ }^{*}$ \\
\hline C. glabrata & $\mathrm{Sal} I^{* *}$ & I a R $R^{\&}$ & $S$ & S \\
\hline C. tropicalis & $\mathrm{S}$ & $S$ & $S$ & $\mathrm{~S}$ \\
\hline C. krusei & $\mathrm{Sal} \mathrm{I}^{* *}$ & $\mathrm{R}^{\wedge}$ & S & $S$ \\
\hline C. lusitaniae & I a R & $\mathrm{S}$ & $S$ & $S$ \\
\hline
\end{tabular}




\section{Endoftalmitis}

Puede ocurrir desde el inicio de la infección fúngica pero es más frecuente en candidemias persistentes. Se ha descrito el compromiso ocular en 3,2\% de las candidemias $^{56}$. El tratamiento recomendado es sistémico, con anfo B-d o anfo-L. Va a depender de si existe sólo corioretinitis, si la infección compromete o no la mácula y el grado de extensión hacia el humor vítreo. El paciente con endoftalmitis requiere evaluación periódica por oftalmólogo. El tiempo de tratamiento depende de la resolución clínica de las lesiones, con un plazo mínimo de 6 semanas.

\section{SNC}

Está presente en $50 \%$ de las candidemias en RNpret extremo ${ }^{11}$. Habitualmente no se asocia a síntomas ni signos neurológicos por lo que el alto índice de sospecha en estos casos es fundamental. Debe tenerse presente que el compromiso de SNC puede presentarse sin alteraciones en el LCR. El tratamiento de elección es anfo B-d o anfo-L, los que alcanzan adecuadas concentraciones en el LCR. El tratamiento se mantiene por 21 días o más, controlando la negativización del cultivo de LCR -en caso de ser inicialmente positivo- a los 7-14 días ${ }^{57}$. De no producirse su negativización es recomendable asociar otro antifúngico con distinto mecanismo de acción, como fluconazol o 5-fluocitocina (este último no está disponible en Chile).

En suma, el tratamiento debe mantenerse hasta que las imágenes patológicas cerebrales presentes al diagnóstico se hayan resuelto, el LCR normalizado y el paciente esté clínicamente asintomático ${ }^{25}$. Las imágenes cerebrales patológicas deben controlarse mediante ecografía cerebral a las dos semanas de iniciar tratamiento y una vez al mes hasta su resolución.

No se recomienda utilizar equinocandinas en las CI con localización encefálica.

\section{Renal}

La infección por Candida spp del tracto genitourinario se considera como una infección invasora en RNpret MBPN por lo que se debe realizar una evaluación exhaustiva de diseminación hacia otro órgano blanco ${ }^{58}$. Se han encontrado alteraciones en la ecografía renal (bolas fúngicas o infiltración del parénquima renal) en $5 \%$ de los casos de candidemia. Un estudio ultrasonográfico inicial normal no descarta la aparición de bolas fúngicas en estudios posteriores, por lo que se recomienda seguir a estos pacientes hasta la resolución del cuadro clínico ${ }^{59}$. El tratamiento de las bolas fúngicas debe considerar un plazo mínimo de terapia antifúngica de cuatro semanas y mantener hasta la completa resolución de las imágenes renales o evidenciar su calcificación.

\section{Osteomielitis}

Complicación poco frecuente en candidemia. Se sospecha clínicamente por edema y/o disminución de la movilidad de una extremidad (al igual que las infecciones osteo-articulares bacterianas). El diagnóstico definitivo se hace a través del hallazgo de Candida spp en el examen directo y/o cultivo de la punción ósea o articular.

La radiografía de huesos largos y la ecotomografía articular son técnicas útiles, no invasoras y de fácil acceso, que apoyan el diagnóstico. El edema de tejidos blandos profundos alrededor de la lesión ósea, se observa aproximadamente al tercer día de evolución en la radiografía (a diferencia de los niños mayores). La cintigrafía se desaconseja en neonatos por su baja especificidad debido a la intensa actividad osteoblástica antes de los 2 meses de edad.

Su tratamiento requiere de cirugía y terapia antifúngica prolongada, durante 4 a 6 semanas, con anfo B-d, anfo-L o equinocandinas ${ }^{11}$.

\section{Seguimiento}

Frente a un HC positivo para Candida spp., se recomienda iniciar tratamiento antifúngico con un medicamento fungicida en forma inmediata: anfo B-d, anfo-L o equinocandinas (estos últimos si se descarta compromiso del SNC) y concomitantemente realizar una evaluación exhaustiva en búsqueda de EFI. Se recomienda además retirar el CVC lo más precozmente posible, idealmente antes de las primeras $24 \mathrm{~h}$. A las $72 \mathrm{~h}$ de iniciar el tratamiento antifúngico se deben controlar los HCs y repetir cada $48 \mathrm{~h}$ hasta tener dos HCs consecutivos negativos. La Figura 1 presenta un algoritmo de seguimiento de candidemia neonatal.

Si en la evaluación inicial, sólo existe aislamiento de Candida spp en sangre y el paciente se encuentra clínicamente estable, el tratamiento se prolonga por 14 días, pudiendo cambiar la terapia a fluconazol (si la cepa aislada es sensible) luego de completar siete días de tratamiento con un medicamento fungicida.

Si por el contrario, el paciente presenta una evolución clínica desfavorable y/o permanecen los HCs positivos al $7^{\circ}$ día, se debe reevaluar la presencia de EFI con los exámenes ya descritos, además de la búsqueda de un foco óseo secundario. Se debe rescatar el estudio de susceptibilidad in vitro de la cepa aislada para evaluar la necesidad de un cambio de terapia antifúngica.

Otro escenario posible es que el paciente mantenga HCs positivos luego de $72 \mathrm{~h}$ de tratamiento antifúngico, lo que se define como una candidemia persistente. En estos casos, debe buscarse nuevamente una localización secundaria mediante un examen clínico detallado, fondo de ojo, exámenes generales, estudio microbiológico y de imágenes. Mientras se realizan los exámenes se debe mantener la terapia antifúngica, evaluar la susceptibilidad in vitro de la cepa aislada y repetir los HCs cada $48 \mathrm{~h}$ hasta 
certificar su negativización. Una vez que el paciente tiene dos HCs consecutivos negativos, se recomienda mantener la terapia por 14 a 21 días. Si el paciente presenta compromiso de algún órgano específico, el tratamiento y otras medidas terapéuticas se ajustarán a cada situación clínica. (Ver Tratamiento de las complicaciones).

\section{Profilaxis}

El diagnóstico de CI puede resultar difícil, ya que las manifestaciones clínicas en RN son inespecíficas, lo que se asocia a la baja sensibilidad de las pruebas diagnósticas. Por eso se ha evaluado ampliamente en la literatura médica la indicación de profilaxis antifúngica en $\mathrm{RN}$, especialmente los RNpret extremos, grupo que reúne la mayor cantidad de factores de riesgo de CI. Estos estudios han demostrado que la profilaxis con fluconazol disminuye la colonización y las CI pero no está claro aún su rol en la disminución de la mortalidad asociada a CI.

Es fundamental evaluar el impacto de la profilaxis en $\mathrm{RN}$ dependiendo de la tasa de incidencia de CI en el grupo de mayor riesgo que son los RNpret extremos, observándose un mayor impacto en aquellas UCIN con tasas de incidencia altas $(\geq 5 \%)$. En UCIN con tasa de incidencia baja e intermedia, es necesario considerar los factores de riesgo presentes y el número necesario a tratar. La literatura científica describe que en UCIN con bajas

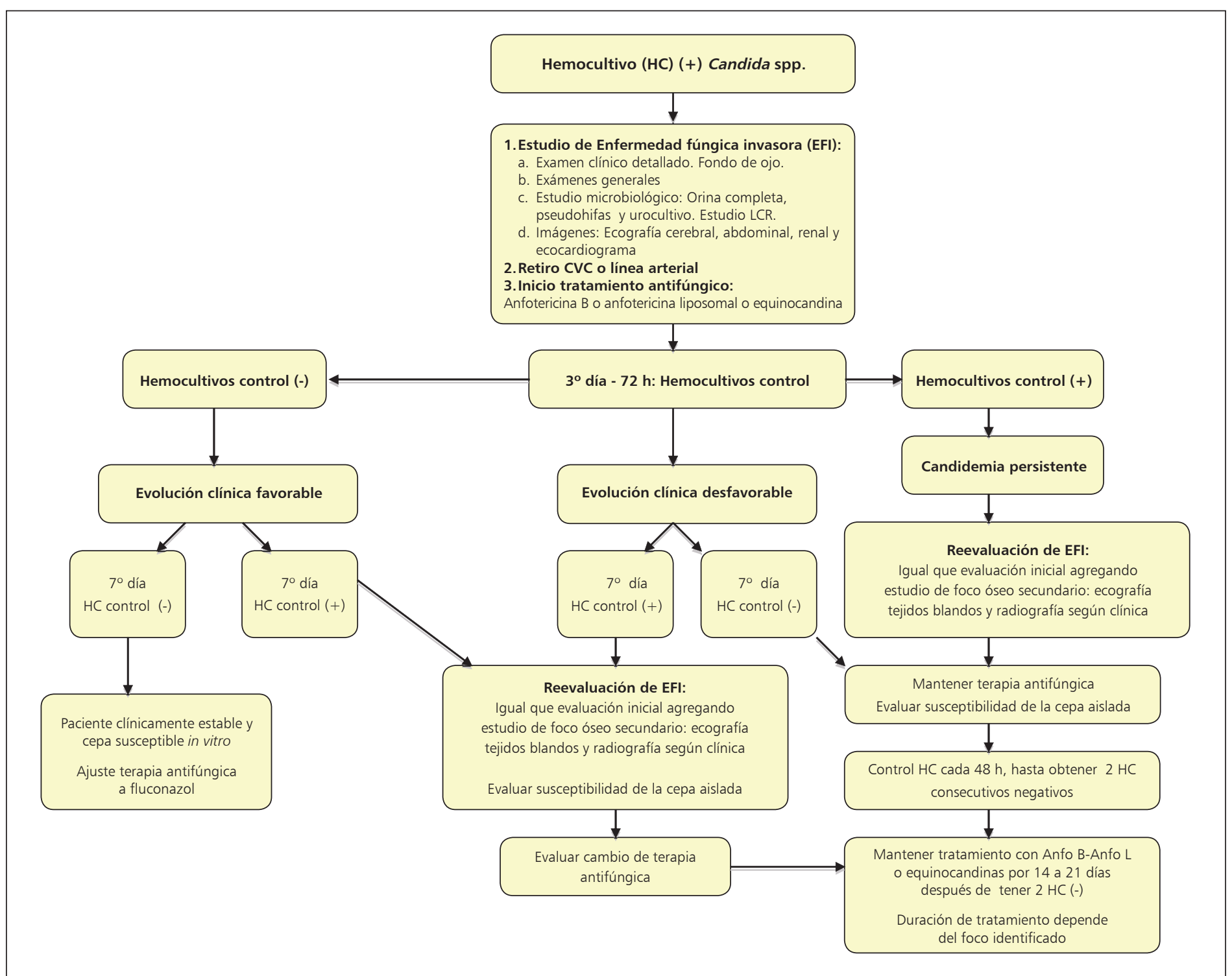

Figura 1. Algoritmo de seguimiento de candidiasis invasora neonatal. 
tasas de incidencia se debe exponer un gran número de $\mathrm{RN}$ a profilaxis con fluconazol (100) para evitar un caso de $\mathrm{CI}^{60}$ (Figura 2).

Kauffman y cols. ${ }^{61}$, observaron en RNpret extremo una disminución de la incidencia de CI desde $20 \%$ en el grupo placebo a $0 \%$ en el grupo con profilaxis. Este estudio se realizó en un solo centro, con una alta incidencia de CI. Revisiones sistemáticas posteriores ${ }^{62,63}$ han demostrado una disminución de CI en RNpret extremo sometidos a profilaxis con fluconazol del orden de $90 \%$ (OR 0,09; IC $95 \% 0,04-0,24 ; \mathrm{p}=0,004)$, con un efecto significativo sobre la mortalidad atribuible a CI (OR 0,04; IC 95\% $0,01-0,31 ; p=0,006)^{64}$.

El principal punto de conflicto de la profilaxis con fluconazol en neonatos de riesgo es si esta medida podría producir cambios epidemiológicos aumentando el porcentaje de aislados de Candida spp. diferentes a $C$. albicans, lo que se relacionaría con aumento de resistencia a fluconazol, tema que permanece en duda y que no se ha confirmado ni descartado hasta ahora.

Nistatina fue el primer antifúngico evaluado para profilaxis en $\mathrm{RN}$ de riesgo de CI. Un estudio comparativo de fluconazol versus nistatina oral como estrategia de profilaxis demostró superioridad de fluconazol en la prevención de CI. Además se describen más efectos adversos con el uso de nistatina oral, debido a la alta osmolaridad observada y un mayor riesgo de enterocolitis necrosante ${ }^{65}$.

Otros potenciales agentes para uso de profilaxis son los probióticos (Lactobacillus reuteri o Lactobacillus rhamnosus) que han mostrado reducir fundamentalmente la colonización intestinal por Candida spp. y la lactofe-

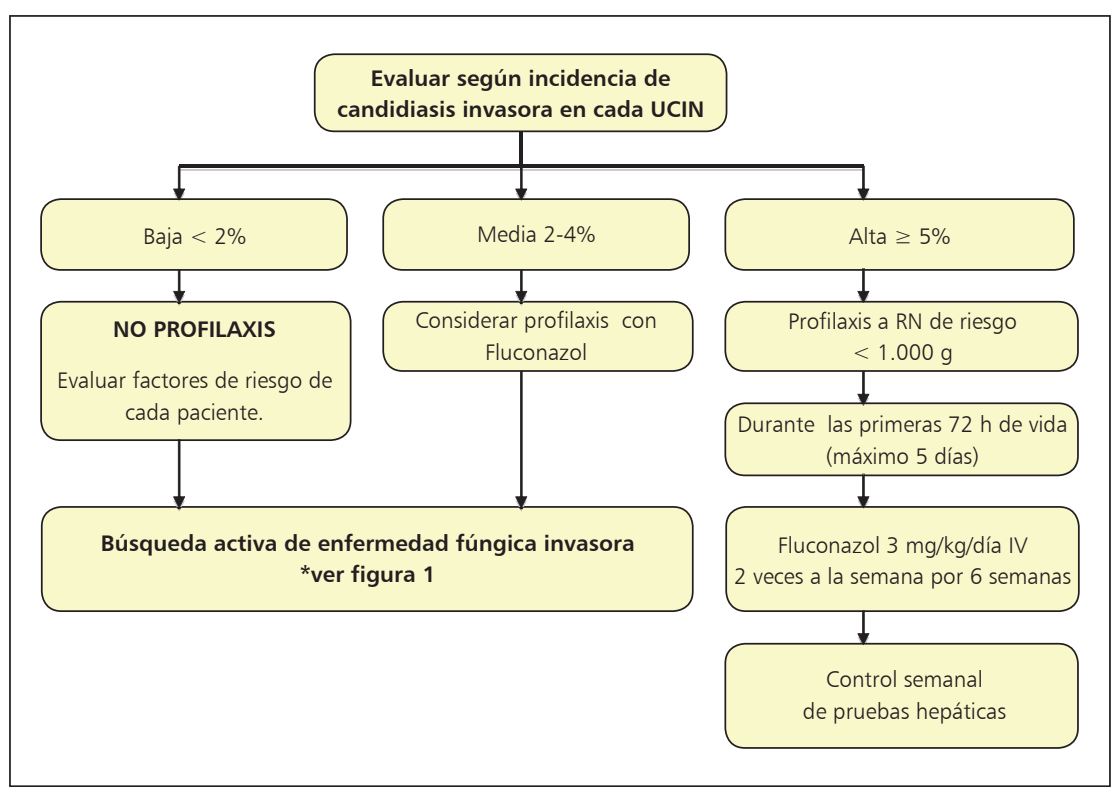

Figura 2. Algoritmo de profilaxis de candidiasis invasora neonatal. rrina bovina que no disminuye la colonización, pero si demostró tener un efecto en la reducción de CI comparado con un grupo control con placebo ${ }^{66}$. Lactoferrina tendría un efecto fungicida sobre Candida spp., sería más fisiológico que el uso de un antifúngico y tendría menos efectos adversos. Faltan estudios que comparen el uso de fluconazol y lactoferrina bovina en la reducción de CI. El uso de probióticos en RN prematuros es una herramienta que parece promisoria en la prevención de enterocolitis necrosante, pero los estudios publicados en la literatura científica varían, tanto en la especie de probiótico como en la dosis y el tiempo de uso ${ }^{67}$. Por ahora parece prudente esperar mayores estudios de riesgo/beneficio antes de instaurar su uso generalizado.

En conclusión, hasta ahora el medicamento recomendado para profilaxis en RNpret extremo y en UCIN con alta tasa de CI es fluconazol, debido a su adecuada farmacocinética y farmacodinamia en RN y buen perfil de seguridad. La dosis es de $3 \mathrm{mg} / \mathrm{kg} /$ día IV iniciado antes de los primeros 5 días de vida, dos veces a la semana, hasta las 6 semanas de vida, o hasta no requerir una vía venosa periférica o central. Se debe monitorizar semanalmente la función hepática ${ }^{63}$ (Tabla 6).

El grupo de expertos de ESCMID recomienda el uso de fluconazol como profilaxis en las UCIN con alta incidencia de $\mathrm{CI}(>5 \%)$. En aquellas unidades donde la incidencia es baja $(<2 \%)$ la sugerencia es realizar una evaluación de cada caso en particular según los factores de riesgo que posea el $\mathrm{RN}^{38}$, teniendo una consideración especial el grupo de RNpret extremo con alta tasa de colonización $(\geq 3 \text { sitios anatómicos })^{68}$.

\section{Vigilancia de colonización por Candida spp.}

Como se ha señalado previamente, la colonización por Candida spp es una de las principales variables a considerar en la progresión a CI ${ }^{19}$, en especial cuando se aísla Candida spp en $\geq 3$ sitios. Los cultivos de vigilancia permiten describir y monitorizar cambios en la epidemiología local (C. albicans vs no-albicans y su patrón de susceptibilidad in vitro a antifúngicos), determinar sitios de colonización más frecuentes y a su vez la intensidad de colonización.

Recomendamos realizar cultivos semanales de la nasofaringe (o secreción endotraqueal en paciente en $\mathrm{VM}$ ), recto y piel (axilas o ingle) en especial en los RNpret extremos.

La adquisición de Candida spp también puede ser por vía horizontal ${ }^{11}$, es decir, a través de contacto directo e indirecto e infección cruzada con personal de salud. Sin embargo, no existen por el momento evidencias que respalden o rechacen el uso de medidas de aislamiento, más allá de las precauciones estándares. Es fundamental un adecuado higiene de manos además del uso racional y juicioso de antimicrobianos en la UCIN ${ }^{69}$. 


\section{Conclusiones}

Las infecciones invasoras por Candida spp en RN son una importante causa de morbi-mortalidad en UCIN que presentan alta incidencia de infecciones fúngicas. En unidades de baja incidencia de CI, como lo es nuestro centro, se requiere de un alto índice de sospecha de EFI, en especial por la baja especificidad de sus síntomas.

Frente a un RN con factores de riesgo de CI, se recomienda iniciar una búsqueda activa de infección por Candida spp con estudio de todos los órganos blanco. Al confirmar una CI, se debe proporcionar un tratamiento antifúngico apropiado de manera inmediata con un fármaco fungicida y retirar antes de $24 \mathrm{~h}$ todos los dispositivos endovasculares que tenga el RN.

El uso de profilaxis con fluconazol sigue siendo controversial en unidades de baja incidencia de CI debido al bajo impacto que éste pudiese tener. Pese a la baja incidencia de EFI en nuestro centro, se recomienda que los equipos tratantes evalúen cada caso en particular y consideren el uso de profilaxis con fluconazol, particularmente en los $\mathrm{RN}$ de menos de $1.000 \mathrm{~g}$ sometidos a cirugía abdominal, con resección de asas, usuarios de NP y con colonización de tres o más sitios anatómicos.

Los mensajes esenciales de este manuscrito a todas las unidades que tratan RN prematuros son dos; por un lado la relevancia de la vigilancia activa de las CI en sus unidades de trabajo, a fin de conocer la incidencia local,

\begin{tabular}{|lccc|}
\hline \multicolumn{4}{|c|}{ Tabla 6. Profilaxis con fluconazol en $<1.000 \mathrm{~g}$ y UCIN con alta tasa } \\
de incidencia de candidiasis invasora $(\geq 5 \%)$
\end{tabular}

las especies involucradas y su susceptibilidad in vitro a los diferentes antifúngicos, y por otro, la búsqueda activa de candidemia en los RN de riesgo. Ambas acciones están dirigidas a adoptar de manera oportuna las mejores decisiones terapéuticas en beneficios de los pacientes.

\section{Resumen}

Las infecciones fúngicas invasoras se han convertido en un problema cada vez más frecuente en las unidades de cuidados intensivos neonatales. La candidiasis invasora (CI) es una infección que se asocia a una significativa morbi-mortalidad, en especial en los recién nacidos ( $\mathrm{RN}$ ) prematuros. El objetivo de este documento es sugerir acciones de vigilancia, prevención farmacológica, diagnóstico, tratamiento y seguimiento de las CI en el RN.

\section{Referencias bibliográficas}

1.- Pooli L, Nocetti Fasolino M, Pereda R, Rial MJ, Califano G. Candidemia en una unidad de cuidados intensivos neonatales: identificaciones de factores de riesgo. Arch Argent Pediatr 2006; 104 (5): 393-8.

1a.- Santolaya ME, Alvarado T, Queiroz-Telles F, Colombo AL, Zurita J, Tiraboschi IN, et al. Active surveillance of candidemia in children from Latin America: a key requirement for improving disease outcome. Pediatr Infect Dis J 2013; August 29 (Epub ahead of print)

2.- Ministerio de Salud (MINSAL). Guía Clínica Prevención Del Parto Prematuro $1^{\circ} \mathrm{Ed}$. Santiago: MINSAL, 2005.

3.- Neu N, Malik M, Lunding A, Whittier S, Alba L, Kubin C, et al. Epidemiology of candidemia at childrens hospital, 2002 to 2006. Pediatric Infect Dis J 2009; 28: 806-9.

4.- Natarajan G, Lulic-Botica M, Rongkvilit C, Pappas A, Bedard M. Experience with caspofungin in the treatment of persistent fungemia in neonates. J Perinatol 2005: 25: 770-7.

5.- Spiliopolou A, Dimitriou G, Jelastopulu E, Giannakopoulus I, Anastassiou E,
Christofidou M. Neonatal intensive care unit candidemia: epidemiology, risk factors, outcome, and critical review of published case series. Mycopathologia 2012; 173: 219-28.

6.- Fridkin S, Kaufman D, Edwards J, Shetty S, Horan T. Changing incidence of Candida bloodstream infections among NICU patients in the United States: 1995-2004. Pediatrics 2006; 117: 1680-7.

7.- Stoll B, Hansen N, Fanaroff A, Wright L, Carlo W, Ehrenkranz R, et al. Late-onset sepsis in very low birth weight neonates: the experience of the NICHD Neonatal Research Network. Pediatrics 2002; 110 (2): 285-91.

8.- Benjamin D, Stoll B, Fanaroff A, McDonald S, Oh W, Higgins RD, et al. Neonatal candidiasis among extremely low birth weight infants: risk factors, mortality and neurodevelopmental outcomes at 18-22 months. Pediatrics 2006; 117 (1): 84-92

9.- $\quad$ Stoll B, Hansen N, Adams-Chapman I, Fanaroff AA, Hintz S, Vohr B, et al. National Institute of Health and Human Development Neonatal Research Network. Neurodevelopmental and growth impairment among extremely low-birth-weight infants with neonatal infection. JAMA 2004; 292 (19):
2357-65.

10.- Tezer H, Canpolat F, Dilmen U. Invasive fungal infections during the neonatal period: diagnosis, treatment and prophylaxis. Expert Opin Pharmacother 2012; 13 (2): 193-205.

11.- Bendel C. Candidiasis. En: Infectious Disease in the Fetus and Newborn, $7^{\text {th }}$ ed, 2010. Remington J, Klein J, Wilson C, Nizet V, Maldonado Y., Eds. Elsevier Saunders, Philadelphia; p. 1055-77.

12.- Hernández-Castro R, Arroyo-Escalante $S$, Carrillo-Casas E, Moncada-Barrón D, Álvarez-Verona E, Hernández-Delgado L, et al. Outbreak of Candida parapsilosis in a neonatal intensive care unit: a health care workers source. Eur J Pediatr. 2010; 169 (7): 783-7.

13.- van Asbeck E, Huang Y, Markham A, Clemons K, Stevens D. Candida parapsilosis fungemia in neonates: genotyping results suggest healthcare workers hands as source, and review of published studies. Mycopathologia. 2007; 164 (6): 287-93.

14.- Santolaya ME, de Queiroz Telles F, AlvaradoMatute T, de Queiroz-Telles F, Colombo AL, Zurita J, et al. Recomendaciones para el manejo de la candidemia en neonatos en América Latina. Rev Iberoam Micol 2013 
Jul-Sep; 30(3 Suppl 1):158-70. doi: 10.1016/j. riam.2013.06.002. Epub 2013 Jun 11.

15.- Steibach W, Roilides E, Berman D, Hoffman J, Groll A, Bin-Hussain I, et al. Results from a prospective, international, epidemiologic study of invasive candidiasis in children and neonates. Pediatr Infect Dis J 2012; 31 (12): 1252-7.

16.- Baley J, Kliegman R, Boxerbaum B, Fanaroff A. Fungal colonization in the very low birth weight infant. Pediatrics. 1986; 78: 225-32.

17.- Huang Y, Li C, Lin T. Association of fungal colonization and invasive disease in very low birth weight infants. Pediatr Infect Dis J 1998; 17: 819-22.

18.- Manzoni P, Arisio R, Mostert M, Leonessa M, Farina D, Latino M, et al. Prophylactic fluconazole is effective in preventing fungal colonization and fungal systemic infections in preterm neonates: a single-center, 6-year, retrospective cohort study. Pediatrics 2006; 117 (1): e22-32.

19.- Manzoni P, Farina D, Leonessa M, Antonielli d'Oulx E, Galletto P, Mostert M, et al. Risk factors for progression to invasive fungal infection in preterm neonates with fungal colonization. Pediatrics 2006; 118 (6): 2359-64.

20.- Caviedes P, Delpiano L, Barraza B, Cifuentes M. Colonización por Candida spp. en recién nacidos menores de 1500 gramos hospitalizados en UCIN. XVI Congreso Panamericano de Infectología y XXX Congreso Chileno de Infectología. Comunicación Oral, CO-22. Mayo 2013, Santiago, Chile.

21.- Shetty S, Harrison L, Hajjeh R, Taylor T, Mirza S, Schmidt A, et al. Determining risk factors for candidemia among newborn infants from population-based surveillance. Baltimore, Maryland, 1998-2000. Pediatr Infect Dis J 2005; 24: 601-4.

22.- Dyke MP, Ott K. Severe thrombocytopenia in extremely low birth weight infants with systemic candidiasis. J Paediatr Child Health. 1993; 29 (4): 298-301

23.- Tirodker UH, Nataro JP, Smith S, LasCasas L, Fairchild KD. Detection of fungemia by polymerase chain reaction in critically ill neonates and children. J Perinatol 2003; 23 (2): 117.

24.- Wellinghausen N, Siegel D, Winter J, Gebert S. Rapid diagnosis of candidaemia by real-time PCR detection of Candida DNA in blood samples. J Med Microbiol 2009; 58: 1106-11.

25.- Pappas P, Kauffman C, Andes D, Benjamin D, Calandra T, Edwards J, et al. Clinical practice guidelines for the management of candidiasis: 2009 update by the Infectious Diseases Society of America. Infectious Diseases Society of America. Clin Infect Dis 2009; 48 (5): 503-35.

26.- Baley J, Meyers C, Kliegman R, Jacobs M, Blumer J. Pharmacokinetics outcome of treatment, and toxic effects of amphotericin
B and 5-fluorocytosine in neonates. J Pediatr 1990; 116: 791-7.

27.- Bes D, Sbernaa N, Rosanovaa MT. Ventajas y desventajas de los distintos tipos de anfotericina en pediatría: revisión de la bibliografía. Arch Argent Pediatr 2012; 110 (1): 46-51.

28.- Turkova A, Roilides E, Sharland M. Amphotericin B in neonates: deoxycholate or lipid formulation as first-line therapy-is there a 'right' choice? Curr Opin Infect Dis 2011, 24: 163-71.

29.- Lee J, Adler-Sohohet F, Nguyen C, Lieberman J. Nephrotoxicity associated with amphotericin B deoxycholate in neonates. Pediatr Infect Dis J 2009; 28(12): 1061-3.

30.- Testoni D, Smith B, Benjamin D. The use of antifungal therapy in neonatal intensive care. Clin Perinatol 2012; 39: 83-98.

31.- López J, Coto G, Fernández B. Neonatal invasive candidiasis: a prospective multicenter study of 118 cases. Am J Perinatol 2003; 20 (3): 153-63.

32.- Altamirante B, Rodriguez D. Antifungal agents in neonates: issues and recommendations. Pediatr Drugs 2007; 9 (5): 311-21.

33.- Queiroz-Tellez F, Berezin E, Levenger G, Freire A, van der Vyver A, Chotpitasunondh T, et al. Micafungin versus liposomal amphotericin $\mathrm{B}$ for pediatric patients with invasive candidiasis: study of a randomized double-blind trial. Pediatr Infect Dis J 2008; 27 (9): 820-6.

34.- Jeon G, Koo S, Lee J, Hwang J, Kim S, Lee E, et al. A comparison of AmBisome to amphotericin B for treatment of systemic candidiasis in very low birth weight infants. Yonsei Med J 2007; 48 (4): 619-26.

35.- Linder N, Klinger G, Shalit I, Levy I, Ashkenazi S, Haski G, et al. Treatment of candidaemia in premature infants: comparison of three amphotericin B preparations. J Antimicrob Chemother 2003; 52 (4): 663-7.

36.- Scarcella A, Pasquariello M, Giugliano B, Venemmia M, De Lucia A. Liposomal amphotericin B treatment for neonatal fungal infections. Pediatr Infect Dis J 1998; 17 (2): 146-8.

37.- Juster-Reicher A, Flidel-Rimon O, Amitay M, Even-Tov S, Shinwell E, Leibovitz E. Highdose liposomal amphotericin B in the therapy of systemic candidiasis in neonates. Eur J Clin Microbiol Infect Dis 2003; 22 (10): 603-7.

38.- Hope W, Castagnola E, Groll A, Roilides E, Akova M, Arendrup MC, et al. ESCMID guideline for the diagnosis and management of Candida disease 2012: prevention and management of invasive infections in neonates and children caused by Candida spp. Clin Microbiol Infect 2012; 18 (S7): 38-52.

39.- Anaissie E, Mattiuzzi G, Miller C, Noskin G, Gurwith M, Mamelok R, et al. Treatment of invasive fungal infections in renally impaired patients with amphotericin B colloidal dispersion. Antimicrob Agents Chemother 1998; 42 (3): 606-11.

40.- White M, Bowden R, Sandler E, Graham M, Noskin G, Wingard J, et al. Randomized, double blind clinical trial of amphotericin B colloidal dispersion vs. amphotericin $\mathrm{B}$ in the empirical treatment of fever and neutropenia. Clin Infect Dis 1998; 27 (2): 296-302.

41.- Wahab Mohamed W, Ismail M. A randomized, double blind, prospective study of caspofungin vs amphotericin B for the treatment of invasive candidiasis in newborns infants. J Trop Pediatr 2012; 58 (1): 25-30

42.- Sáez-Llorens X, Macias M, Maiya P, Pineros J, Hafri H, Chatterjee A, et al. Pharmacokinetics and safety of caspofungin in neonates and infants less than 3 months of age. Antimicrob Agents Chemother 2009; 53: 869-75.

43.- Odio C. Caspofungin (CAS) therapy (Tx) for neonates (Ns) with invasive candidiasis (IC) cared for at intensive care units (ICU). 46th Interscience Conference on Antimicrobial Agents and Chemotherapy (ICAAC). San Francisco, September 27-30, 2006. Abstract G-976.

44.- Odio C. Terapia antifúngica para neonatos, niños y adolescentes con micosis invasiva sospechada o documentada. Drugs of Today 2010; 46: 33-46.

45.- Queiroz-Telles F, Berezin E, Leverger G, Freire A, van der Vyver A, Chotpitayasunondh T, et al. Micafungin Invasive Candidiasis Study Group. Micafungin versus liposomal amphotericin B for pediatric patients with invasive candidiasis: substudy of a randomized double-blind trial. Pediatr Infect Dis J 2008; 27 (9): 820-6.

46.- Hope W, Smith B, Arrieta A, Buell D, Roy M, Kaibara A, et al. The pharmacokinetics and pharmacodynamics of micafungin in experimental hematogenous Candida meningoencephalitis: Implications for echinocandin therapy in neonates. J Infect Dis 2008; 197:163-71.

47.- Catalán-González M, Montejo-González J. Farmacodinamia y farmacocinética de la micafungina en adultos, niños y neonatos. Rev Iberoam Micol 2009; 26 (1): 23-34.

48.- Tripathi N, Watt K, Benjamin D. Treatment and prophylaxis of invasive candidiasis. Semin Perinatol 2012; 36 (6): 416-23.

49.- Arrieta A, Maddison P, Groll A. Safety of micafungin in pediatric clinical trials. Pediatr Infect Dis J 2011; 30(6): 97-102.

50.- Varisco B, Benner K, Prabhakaran P. Neonatal peritoneal candidiasis successfully treated with anidulafungin add-on therapy. Ann Pharmacother 2009; 43 (11): 1907-10.

51.- Procianoy RS, Silveira RC. Epidemiology of candidemia. J Clin Microbiol 2008; 46(5): 1894.

52.- Turner K, Manzoni P, Benjamin D, CohenWolkowiez M, Smith P, Laughon M. Fluconazole pharmacokinetics and safety in 
premature infants. Curr Med Chem 2012; 19 (27): 4617-20.

53.- Piper L, Smith P, Hornik C, Cheifetz I, Barrett J, Moorthy G, et al. Fluconazol loading dose pharmacokinetics of fluconazole in young infants. Pediatr Infect Dis J 2011; 30 (5): 375-8.

54.- Benjamin D, Ross K, McKinney R, Benjamin D, Auten R, Fisher R. When to suspect fungal infection in neonates: a clinical comparision of Candida albicans and Candida parapsilosis fungemia with coagulase-negative staphylococcal bacteremia. Pediatrics 2000; 106: 712-8.

55.- Levy I, Shalit I, Birk E, Sirota L, Ashkenazi S, German B, et al. Candida endocarditis in neonates: report of five cases and review of the literature. Mycoses 2006; 49(1): 43-8.

56.- Fierro J, Prasad P, Fisher B, Gerber J, Coffin S, Walsh T, et al. Ocular manifestations of candidemia in children. Pediatr Infect Dis J 2013; 32 (1): 84-6.

57.- Voice R, Bradley S, Sangeorzan J, Kauffman C. Chronic candidal meningitis: an uncommon manifestation of candidiasis. Clin Infect Dis
1994; 19(1): 60-6.

58.- Wynn J, Tan S, Gantz M, Das A, Goldberg R, Adams-Chapman I, et al. Outcomes following candiduria in extremely low birth weight infants. Clin Infect Dis 2012; 54 (3): 331-9.

59.- Noyola D, Fernandez M, Moylett E, Baker C. Ophthalmologic, visceral, and cardiac involvement in neonates with candidemia. Clin Infect Dis 2001; 32 (7): 1018

60.- Benjamin D. First, Do no harm. Pediatrics 2008; 121 (4): 831-2.

61.- Kauffman D, Boyle R, Hazen K, Patrie J, Robinson M, Donowitz L. Fluconazole prophylaxis against fungal colonization and infection in preterm infants. N Engl J Med 2001; 107: 293-8.

62.- Kauffman D. Prevention of invasive candida infections in preterm infants: the time is now. Expert Rev Anti Infect Ther 2008; 6: 393-9.

63.- Kauffman D. Neonatal candidiasis: clinical manifestations, management and prevention strategies. J Pediatr 2010; 156 (4): 53-66.

64.- Manzoni P. The use of fluconazole in NICU. Arch Dis Child 2009; 94: 983-7.
65.- Violaris K, Carbone T, Bateman D, Olawepo O, Doraiswamy B, LaCorte M. Comparison of fluconazol and nystatin oral suspensions for prophylaxis of systemic fungal infection in very low birth weight infants. Am J Perinatol 2010; 27: 73-8.

66.- Manzoni P, Stolfi I, Cattani S, Laforgia N, Romeo M, Bollani L, et al. Bovine lactoferrin prevents invasive fungal infections in very low birth weight infants: a randomized controlled trial. Pediatrics 2012; 129: 116-23.

67.- Caffarelli C, Bernasconi S. Preventing necrotizing enterocolitis with probiotics. Lancet 2007; 369 (9573): 1578-80.

68.- Manzoni P, Mostert M, Jacqz-Aigrain E, Stronati M, Farina D. Candida colonization in the nursery. J Pediatr (Rio J) 2012; 88 (3): 187-90.

69.- Mohan P, Eddama O, Weisman LE. Patient isolation measures for infants with candida colonization or infection for preventing or reducing transmission of candida in neonatal units. Cochrane Database Syst Rev 2007; 3: CD006068. 